\title{
Interpretation of plasma amino acids in the follow-up of patients: The impact of compartmentation
}

\author{
Claude Bachmann
}

Received: 18 September 2007 /Submitted in revised form: 7 December 2007 / Accepted: 12 December 2007 /

Published online: 31 January 2008

(C) SSIEM and Springer 2007

\begin{abstract}
Summary Results of plasma or urinary amino acids are used for suspicion, confirmation or exclusion of diagnosis, monitoring of treatment, prevention and prognosis in inborn errors of amino acid metabolism. The concentrations in plasma or whole blood do not necessarily reflect the relevant metabolite concentrations in organs such as the brain or in cell compartments; this is especially the case in disorders that are not solely expressed in liver and/or in those which also affect nonessential amino acids. Basic biochemical knowledge has added much to the understanding of zonation and compartmentation of expressed proteins and metabolites in organs, cells and cell organelles. In this paper, selected old and new biochemical findings in PKU, urea cycle disorders and nonketotic hyperglyci-
\end{abstract}

Communicating editor: Georg Hoffmann

Competing interests: None declared

This paper is based in the European Metabolic Group (EMG) Lecture given at the 39th EMG Meeting in Warsaw on 3 June 2007 - organized and sponsored by Milupa Metabolics.

References to electronic databases: Phenylketonuria: OMIM 262600. Urea cycle enzymes, mitochondrial: EC 2.3.1.1, 2.1.3.3, 6.3.4.16. Urea cycle enzymes, cytosolic: EC 3.5.3.1, 4.3.2.1, 6.3.4.5. HHH syndrome: OMIM 238970. Citrin deficiency: OMIM 603859. Lysinuric protein intolerance: OMIM 222700. Branched-chain aminotransferase: EC 2.6.1.42. Non-ketotic hyperglycinaemia: OMIM 605899. Hereditary hyperekplexia: OMIM 149400.

\section{Bachmann}

Clinical Chemistry, University of Lausanne,

Lausanne, Switzerland

C. Bachmann $(\varangle)$

Rittergasse 11, CH 4103 Bottmingen, Switzerland

e-mail: claude.bachmann@gmail.com naemia are reviewed; the aim is to show that integrating the knowledge gained in the last decades on enzymes and transporters related to amino acid metabolism allows a more extensive interpretation of biochemical results obtained for diagnosis and follow-up of patients and may help to pose new questions and to avoid pitfalls. The analysis and interpretation of amino acid measurements in physiological fluids should not be restricted to a few amino acids but should encompass the whole quantitative profile and include other pathophysiological markers. This is important if the patient appears not to respond as expected to treatment and is needed when investigating new therapies. We suggest that amino acid imbalance in the relevant compartments caused by over-zealous or protocol-driven treatment that is not adjusted to the individual patient's needs may prolong catabolism and must be corrected.

$\begin{array}{ll}\text { Abbreviations } \\ \text { IEM } & \text { inborn error of metabolism } \\ \text { PKU } & \text { Phenylketonuria } \\ \text { UC } & \text { urea cycle } \\ \text { UCD } & \text { urea cycle disorder } \\ \text { NKH } & \text { nonketotic hyperglycinaemia } \\ \text { LNAA } & \text { large neutral amino acid }\end{array}$

\section{Introduction}

It is obvious that patients, organs or cells are not homogeneous with respect to protein and metabolite concentrations; knowledge of compartmentation and zonation is implied when in daily practice we use results of enzymes assayed in plasma for medical decisions as indicators of tissue damage (e.g. CK-MB, 
ALAT, ASAT), but the basic knowledge of the tissue expression of these proteins and isoforms is not always consciously kept in mind. In inborn errors of metabolism (IEMs) such knowledge of compartmentation should be taken into account when interpreting concentrations of small molecules such as amino acids, lactate, pyruvate or carnitine(s) because clinical symptoms are not sufficient to allow decisions to be made.

Results of concentration measurements in whole blood are not equal to plasma results even if the solute concentration is the same in the aqueous space of plasma and red cell. This is due to the volume occupied by plasma proteins (and proteins from haemolysed red cells) in the fixed sample volume. In plasma this amounts to $\sim 7 \%$; in whole blood the protein displacement is about $17-19 \%$ of the sample volume at a haematocrit of 0.45 . The amount of solute measured is expressed per sample volume in both instances but the aqueous volume differs. This effect is common knowledge for glucose measurements and different decision limits of glucose concentration are used for diagnosing diabetes depending on the sample analysed $(6.3 \mathrm{mmol} / \mathrm{L}$ in whole blood and $7.0 \mathrm{mmol} / \mathrm{L}$ in plasma). Whole-blood concentrations are thus influenced by the haematocrit. In neonates with a widely variable haematocrit this effect is even more pronounced, a fact well known to the neonatal screening specialists who assume a haematocrit of 0.55 for calibration.

The same principle applies to amino acids and other small molecules; in consequence it is necessary to indicate the sample type (and method) used to allow interpretation and correct comparison with data from the literature including decision or reference limits when reporting results. As for glucose, the time delay between the last food intake and sampling affects the plasma results and must be reported. However, this information is not always given in publications of metabolite results. With acylcarnitines the interpretation is further complicated by the differences of concentration and pattern between plasma and red cell acylcarnitines which contribute to the results obtained from dried blood spots.

For many IEMs, direct extrapolations from plasma concentration of amino acids to the underlying pathogenic process can be misleading if one does not take into account the many mechanisms modifying amino acid concentrations between the location where pathology occurs and the metabolite result in the sample analysed. In the majority of IEMs there are still gaps in knowledge in the vast puzzle of regulatory mechanisms intervening within cells, between cells and between different cell types of organs, leading to a diversity of expression within and between organs. Thus, awareness of basic knowledge in physiological and pathological biochemistry is needed in order to avoid pitfalls and appreciate the limits of interpretation imposed on us when we try to understand patterns of amino acids in plasma or other markers of intermediary metabolism. Recent or sometimes neglected older biochemical findings in classic phenylketonuria (PKU), urea cycle disorders (UCDs) and nonketotic hyperglycinaemia $(\mathrm{NKH})$ are reviewed, and their potential impact is discussed.

\section{Phenylketonuria}

The success of newborn screening by the Guthrie test and dietary restriction of the essential amino acid phenylalanine-complemented by special amino acid mixtures devoid of phenylalanine-has directed research in PKU mainly towards questions related to the optimization and evaluation of treatment. Relatively few questions about phenylalanine toxicity to the brain have been addressed (238 publications on pathophysiology out of 13800 on PKU up to 2007).

Noncompliance with treatment and neurological symptoms in postpubertal patients revived interest in old findings: Using the bolus injection technique Oldendorf and colleagues (Oldendorf et al 1971) had tested an earlier hypothesis (Appel 1966) that there is competition between large neutral amino acids (LNAAs) for brain uptake from the blood. They found that selenomethionine uptake in PKU patients was decreased by high phenylalanine concentrations in plasma. Kinetic data of the L1 transporter that is expressed on the luminal membrane of brain capillary endothelium were later established in non-anaesthetized rats (Miller et al 1985); the data supported Oldendorf's findings.

Large neutral amino acids and phenylalanine in brain

Because neurological symptoms were manifested after discontinuing the diet or because phenylalanine concentration in plasma increased owing to noncompliance in adolescent PKU-patients, the Glostrup group proposed alternative treatments with addition of LNAAs (Güttler and Lou 1986; Lou et al 1985). Following that lead, Pietz and colleagues (1999) investigated the effect of peroral administration of LNAAs on the transport of phenylalanine into the brain in vivo. They administered an acute phenylalanine load 
$(100 \mathrm{mg} / \mathrm{kg})$ to $\mathrm{PKU}$ patients with or without added LNAAs $(150 \mathrm{mg} / \mathrm{kg})$. Brain phenylalanine was quantified in vivo by proton magnetic resonance spectroscopy at 1.5 tesla simultaneously with the sampling for plasma amino acids up to $24 \mathrm{~h}$ post load. In addition, I calculated the uptake velocity from the plasma concentrations of phenylalanine and the other LNAAs competing for the L1 transporter from the published data (Pietz et al 1999). The co-application of LNAAs blocked the post-load rise of the already increased phenylalanine concentrations in vivo in the brain (Table 1) as compared with the increase found in patients without co-application of LNAAs. In contrast to the measured brain concentrations, the calculated uptake velocity of phenylalanine remained excessively high and dropped only slightly on addition of LNAAs.

Interestingly, co-administration of LNAAs consistently prevented the EEG frequency changes (increase of theta activity and decrease of alpha2 activity) found up to $24 \mathrm{~h}$ after load in the PKU patients receiving the phenylalanine load alone. As noted by the authors, the effect of the load on the EEG cannot be explained by the competition for uptake at the blood-brain barrier alone. Brain uptake velocity from an arterial bolus does not equal net transport into and out of the brain or its cellular compartments. Amino acid transporters at the abluminal membrane of the capillary endothelia exporting amino acids out of the brain (see below), and plasma membrane transporters of the glia and neurons modify the net transport of amino acids (for a review see Hawkins et al 2006).
Brain uptake velocity of leucine

In addition to the effect of co-administration of LNAAs on the EEG and on in vivo brain concentrations of phenylalanine, one can further ask whether the coadministration of LNAAs as applied by Pietz and colleagues (1999) normalized the influx of competing LNAAs in the brain and especially of leucine, a highaffinity substrate for the L1 transporter. Recent findings show that leucine plays a role not only as a substrate for protein synthesis but also as a signalling molecule for translation initiation (mTor complex, see below). Taub and Johnson (1975) had observed that phenylalanine injections led to polyribosome disaggregation in the brain in neonatal mice and concluded that 'the monomeric ( $80 \mathrm{~S})$ ribosomes were inactive ... with regard to protein synthesis'.

Current MRS techniques (at $1.5 \mathrm{~T}$ ) are not sensitive enough to measure physiological or even decreased concentrations of LNAAs in the low micromolar range and no in vivo data on the brain concentrations of leucine in PKU patients are available to our knowledge.

Despite the fact that high brain concentrations of phenylalanine correlated poorly with the calculated uptake velocity (Table 1), the uptake velocity of leucine was calculated from the reported amino acid plasma concentrations by using the same kinetic variables (Miller et al 1985) of the L1 system as Pietz and colleagues (1999). The uptake velocity of leucine in PKU patients remained below the control values as shown in Table 1 except when the LNAAs were at peak

Table 1 Concentrations of phenylalanine and leucine in brain and plasma and brain uptake velocity after phenylalanine load in PKU patients

\begin{tabular}{|c|c|c|c|c|c|c|}
\hline \multirow[t]{2}{*}{ Load } & \multirow{2}{*}{$\begin{array}{l}\text { Time post } \\
\text { load }(\mathrm{h})\end{array}$} & \multirow{2}{*}{$\begin{array}{l}\text { MRS brain Phe } \\
(\mu \mathrm{mol} / \mathrm{kg})^{\mathrm{a}}\end{array}$} & Phe & Leu & v-Phe & v-Leu \\
\hline & & & \multicolumn{2}{|c|}{$\overline{\text { Plasma concentration }(\mu \mathrm{mol} / \mathrm{L})^{\mathrm{a}}}$} & \multicolumn{2}{|c|}{$\begin{array}{l}\text { Calculated brain uptake velocity } \\
(\mu \mathrm{mol} / \mathrm{min} \text { per } \mathrm{kg} \text { tissue })^{\mathrm{b}}\end{array}$} \\
\hline Phe & 0 & 252 & 1036 & 136 & 57.2 & 7.0 \\
\hline Phe & 6 & 344 & 1890 & 120 & 95.9 & 5.9 \\
\hline Phe & 12 & 377 & 1838 & 135 & 93.4 & 6.6 \\
\hline Phe & 24 & 397 & 1693 & 109 & 87.1 & 5.4 \\
\hline Phe + LNAAs & 0 & 226 & 1063 & 146 & 58.2 & 7.5 \\
\hline Phe + LNAAs & 6 & 235 & 1887 & 398 & 92.7 & 19.1 \\
\hline Phe + LNAAs & 12 & 210 & 1669 & 338 & 82.7 & 16.3 \\
\hline Phe + LNAAs & 24 & 309 & 1755 & 115 & 88.8 & 5.6 \\
\hline Controls $^{\mathrm{a}}$ & 0 & & $40-70$ & $75-170$ & 6.0 & 9.4 \\
\hline
\end{tabular}

Phe, phenylalanine; Leu, leucine; v, velocity; LNAA, large neutral amino acid.

${ }^{a}$ Data from Pietz et al (1999).

${ }^{\mathrm{b}}$ Calculated brain uptake velocity. 


\section{Postprandial brain uptake velocity of Leucine} in normal babies (age 4 months)

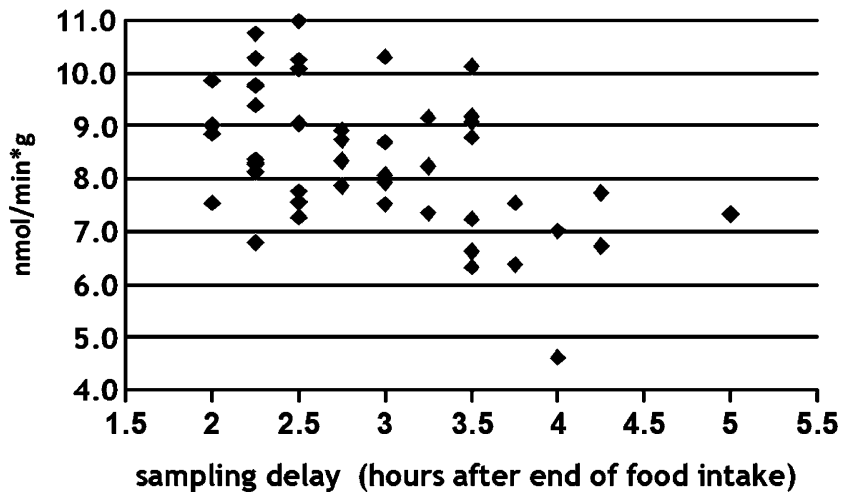

Fig. 1 Brain uptake velocity of leucine calculated from plasma data of normal babies (age 4 months) against the sampling delay after the end of the last food intake

level with leucine concentrations above $340 \mu \mathrm{mol} / \mathrm{L}$ (reference values of healthy adults, 75-170 $\mu \mathrm{mol} / \mathrm{L}$ ). With the average plasma data on 10 adult controls of Pietz and colleagues (1999), an uptake velocity for leucine of $9.4 \mathrm{nmol} / \mathrm{min}$ per $\mathrm{g}$ was obtained. As shown in Fig. 1, the uptake velocity of leucine in normal babies aged 4 months sampled around trough level are mostly above $6 \mathrm{nmol} / \mathrm{min}$ per g). These were computed from amino acid concentrations found in plasma taken at the times indicated (E. Haschke-Becher and C. Bachmann, personal observation). The findings indicate that the LNAA supplements co-administered with the load do not correct the leucine uptake, most likely due to competition by the high plasma phenylalanine concentration, except perhaps at the peak level of LNAAs, when their concentrations are markedly increased.

Accordingly, knowledge of leucine concentration in brain and transport kinetics would be of interest for assessing whether low leucine impairs protein synthesis in the developing brain and contributes to the irreversible symptoms of PKU. Unfortunately, in vivo brain measurements of phenylalanine were not done in a recent pilot experiment of chronic application of LNAAs to PKU (Koch et al 2003). In vitro data of leucine concentrations in brain were not reported for PKU-mice (ENU mutation) fed LNAAs supplements (Matalon et al 2003).

Thus, it remains open whether leucine is decreased in untreated PKU in the brain, and whether long-term LNAA co-supplementation avoids low leucine concentrations in the different cells of the developing brain in PKU which could slow or impair protein synthesis. The use of 7.6 T MRS instruments with animal models might perhaps allow this to be determined in the future.
Recently a commercial product for peroral application of a supplement of LNAAs (and arginine) was evaluated in a pilot experiment on $20 \mathrm{PKU}$ patients (Matalon et al 2006, 2007). The aim was to reduce the uptake of phenylalanine into the intestine. The following transporters are probably involved (Berger et al 2000; Verrey et al 2004): on the apical side of the intestinal epithelia system $b^{0,+}$ and System $\mathrm{B}^{0}$, and on the basolateral side system $y^{+} \mathrm{L}$ and system $\mathrm{L}$; this occurs in conjunction with the exchange of LNAAs with arginine by the cationic amino acid transport systems $b^{0,+}$ and $y^{+}$L. Matalon et al $(2006,2007)$ reported a decline of phenylalanine concentration in plasma of the patients; however, sampling was done on average $2 \mathrm{~h}$ postprandially. The results of these authors merely indicate that the intestinal absorption of phenylalanine could be reduced at around the time of peak absorption of the intestine, but no data were given on trough concentration of phenylalanine or on the area under the time-concentration curve during absorption. No data on the other LNAAs were shown. Thus we cannot estimate whether in humans such a diet will affect transport into the brain and whether, for example, transport of leucine, tryptophan and tyrosine into organs (including the brain) will be sufficient for such a treatment of PKU patients.

\section{Urea cycle disorders}

The follow-up of patients with urea cycle disorders (UCDs) includes frequent monitoring of plasma ammonia and amino acids in order to adapt the treatment to the individual requirements of each patient. In the plasma samples of such patients, glutamine concentrations at the upper limit of reference and low-branched-chain amino acid (BCAA) concentrations are often found. In adults with hepatic insufficiency or cirrhosis, low-branched chain amino acid concentrations in plasma are well known. We will focus on glutamine and its connection to BCAAs.

Glutamine has a central position in intermediary metabolism:

(1) It is a substrate for synthesis of the purines (phosphoribosylpyrophosphate (PRPP) amidotransferase), pyrimidines (carbamylphosphate synthetase 2 trifunctional complex), amino sugars, asparagine, NAD and GMP and thus especially important for tissues with high turnover like the mucosa of the intestinal tract or in cell cultures.

(2) Glutamine synthesis from ammonia and glutamate catalysed by glutamine synthase is active in most major organs; glutamine synthase is not expressed in neurons. Glutamine is a vehicle for 
transporting amino- and amido-nitrogen and fuel between organs, cells and cell organelles and links the Krebs cycle to the urea cycle.

(3) In the liver glutamine synthesis (high affinity but low capacity for ammonia) is restricted to a small pericentral zone of the acini, together with the mitochondrial ornithine aminotransferase and the mitochondrial membrane transporter importing ornithine into the mitochondria in exchange with $\mathrm{H}^{+}$(Dingemanse et al 1996; Gebhardt et al 2007; Häussinger 1983; Jungermann and Kietzmann 1996). In contrast, the three urea cycle enzymes involved in the mitochondrial detoxification of ammonia are located in the periportal zones of the liver acini as well as the cytosolic enzymes of the urea cycle (and the mitochondrial membrane transporter which imports ornithine into the mitochondria in exchange for citrulline). This localization in the entry zone of the portal blood allow detoxification of the bulk of ammonia originating from the splanchnic bed to urea, while the pericentral zone is the second line of defence generating glutamine. Urea cycle enzymes are also expressed in the intestinal tract (at a lower activity than in liver) where postnatally the enzymes mainly catalyse citrulline production from intestinal glutamine. At the cellular level the study of patients with transporter defects (ornithine transporter deficiency leading to $\mathrm{HHH}$-syndrome, citrin deficiency, and lysinuric protein intolerance) has been useful for validating in vitro results of basic research on the affected transporters of the mitochondrial membrane, or the basolateral membrane intestinal and renal epithelia (Palmieri 2004).

(4) The kidney is the main organ for excretion of waste nitrogen, mainly as urea-N (80-90\% of total $\mathrm{N}$ ). Glutamine is excreted into the urine or deaminated to glutamate and ammonia by glutaminase, which is stimulated by acidosis (Nissim 1999; Welbourne 1987). After transport into the renal tubule $\mathrm{NH}_{3}$ is trapped as $\mathrm{NH}_{4}^{+}$in the acidic urine. Argininosuccinate synthetase and lyase are expressed in the cortical part of the kidney tubules while arginase 2 is mainly expressed in the medullary part (Miyanaka et al 1998). This zonation might be relevant for the guanidinoacetate synthesis from arginine and glycine.

\section{Glutamine concentration in plasma}

The glutamine concentration in plasma at steady state results from the sum of cytosolic concentrations in the cells of the respective organs and tissues and from the activity of the glutamine plasma membrane transporters. For each organ and tissue, glutamine concentration depends on its synthesis by glutamine synthase and the cytosolic concentrations of ammonia, glutamate and ATP; on its utilization as substrate; on its transport into the mitochondria; on its breakdown to glutamate (catalysed by glutaminases); and on the transport across the plasma membranes (Curthoys and Watford 1995; Labow et al 2001; Nissim 1999). The major sites for glutamine synthesis are the muscle, lung, liver and adipocytes (Curthoys and Watford 1995). An important proportion of muscle glutamate nitrogen (up to $50 \%$ ) originates from branched-chain amino acids (Chang and Goldberg 1978).

During hyperammonaemia in experimental animals and adult patients the increase in plasma of glutamine parallels the decrease of BCAAs (Leweling et al 1996); this cannot be explained by hyperinsulinism alone, which stimulates the transport of BCAAs into cells and delays the intracellular depletion of BCAAs. The transamination of BCAAs to form glutamate in the mitochondria is catalysed by the branched-chain aminotransferase (mBCAT; muscle $>$ adipose tissue $>$ brain and other tissues). The biochemistry of this enzyme has been studied intensively by the group of Hutson (Bixel et al 2001; Hall et al 1993; Hutson et al 1992, 1998, 2001). mBCAT appears also to stimulate the export of $\alpha$-ketoisocaproate (KICA) out of the mitochondrion, and to maintain a low intramitochondrial concentration of KICA favouring the transamination of leucine. Furthermore, mBCAT forms a supramolecular complex in mitochondria by association with the E1 unit of branched-chain keto acid dehydrogenases, and thus allows channelling of KICA into this irreversible step of BCAA degradation (Islam et al 2007).

Thus when glutamine synthesis is increased in the peripheral organs for ammonia detoxification and/or consumed for conjugation with phenylacetate and phenylbutyrate (in the liver and kidney), more glutamate is utilized; in consequence, BCAA will decrease in the cells and then in plasma.

It has been known for years that acute hyperammonaemia blocks protein synthesis. A possible mechanism has recently been unravelled: leucine is not only an essential substrate for protein synthesis but also a signalling molecule for translation initiation by stimulating mTor (for details see Codogno and Meijer 2005; Harris et al 2006; Meijer and Dubbelhuis 2004; Nishitani et al 2004; Proud 2004a, b; Rennie et al 2004; Yoshizawa 2004). The characteristics have been studied in vitro; the in vivo cellular concentration in 
different human tissues at which leucine limits protein synthesis has not been defined, to our knowledge. It can be speculated that persistently low plasma leucine concentration will prolong catabolism in patients and limit protein synthesis if the tissue concentrations of leucine are chronically decreased.

Another mechanism which might modulate the activity of mTor is mediated by the insulin-like growth factors (IGFs). A leucine-deficient diet leads to decreased free IGF in rats (IGF 1 and 2). The more pronounced expression of the IGF binding protein 1 than of the IGF1 leads to a decrease of the free fractions (Bruhat et al 1999; Endo et al 2002; Jousse et al 1998). These chronic in vivo experiments support the hypothesis that low leucine supply inhibits protein synthesis, because free IGF1 acts finally on mTor via the insulin receptor substrate.

Glutamine metabolism in brain

Compartmentation of glutamine metabolism and transport in brain is rather complex as shown in Fig. 2. Brain expresses a special isoform of glutamate dehydrogenase (encoded by GLUD2) which is more sensitive to leucine activation in presence of ADP than the housekeeping (GLUD1-encoded) enzyme and much less sensitive to GTP inhibition (Plaitakis et al 2003); this might be relevant for understanding the pathogenesis of the hyperinsulinism-hyperammonaemia syndrome.

Another isoform which is mainly expressed in brain concerns BCAA metabolism: In addition to the mitochondrial isoform of BCAT, a second cytosolic isoform (cBCAT) has been characterized (Sweatt et al 2004). This is mainly found in glutamatergic axon terminals of the cortex and also in GABAergic cell bodies; it is inhibited by gabapentin. The glutamine/ glutamate shuttle between astrocytes and neurons is well known (Bak et al 2006). An analogous cycle for branched-chain amino acids has been proposed (Brosnan and Brosnan 2006; Hutson et al 2001; Yudkoff et al 2005). By coupling the two shuttles between astrocytes (mitochondrial BCAT and cytosolic glutamine synthase) and neurons (cytosolic BCAT and kidney type mitochondrial glutaminase), this compartmentation could allow replenishment of the glutamate in the axon terminals after high glutamatergic activity. Moreover, the

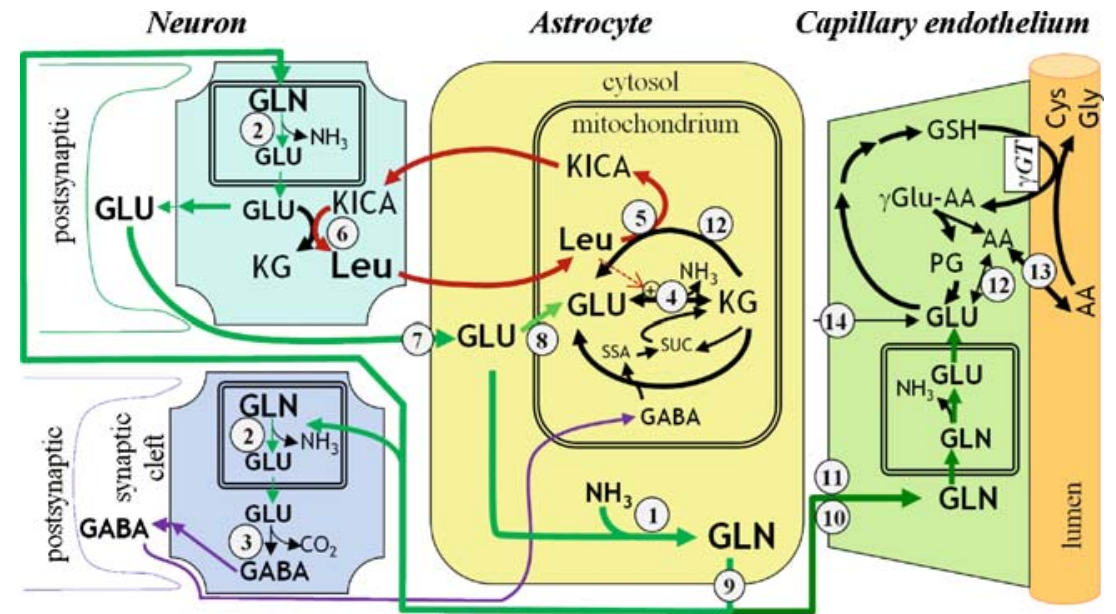

Fig. 2 Simplified scheme of the glutamine/glutamate and leucine shuttles and connection to the brain capillary endothelia. Abbreviations: $\mathrm{NH}_{3}$, ammonia/ammonium; GLU, glutamate; GLN, glutamine; KG, $\alpha$-ketoglutarate; Leu, leucine; KICA, $\alpha$-ketoisocaproate; GABA, $\gamma$-aminobutyrate; SSA, succinate semialdehyde; SUC, succinate (Krebs cycle); $\gamma \mathrm{GT}, \gamma$-glutamyltranspeptidase; GSH, glutathione; PG, pyroglutamate (gamma-glutamyl cycle intermediate); Gly, glycine; Cys, cystein; AA, amino acid. Enzymes and amino acid transporters: 1, Glutamine synthase (cytosolic). 2, Glutaminase (kidney type; inner mitochondial membrane). 3, Glutamate decarboxylase. 4, Glutamate dehydrogenases (mitochondrial); two isoforms: GLUD1 derived , housekeeping enzyme and GLUD2 derived, brain-specific, less sensitive to GTP inhibition, stimulated by lower leucine + ADP concentrations than the housekeeping enzyme; for details see Plaitakis et al (2003). 5, Branched-chain aminotransferase, mitochondrial isoform (mBCAT). 6, Branched-chain aminotransferase, cytosolic isoform (cBCAT) mainly expressed in brain (see text). 7, Glutamate transporters EAAT1 (= GLAST), EAAT2 (= GLT1). 8, Mitochondrial glutamate transporter (inhibited by histidine) (Pichili et al 2007). 9, SNAT3 (= SN1) main glutamine transporter at astrocyte membrane, sodium-dependent. 10, System N subfamily, sodium dependent $($ SNAT3 = SN1). 11, System A transporter, sodium-dependent (SNAT1 $(?=\mathrm{GlnT}$; NAT1). 12, Aminotransferases. 13, Facilitative transporters at the luminal membrane L-system (LAT1). 14, Abluminal glutamate transporters EAAT1-3, sodium-dependent. For further information: on blood-brain barrier transport of amino acids and alias of transporter denomination, see. Chen et al (2004), Hawkins et al (2006), Kanai and Hediger (2004), Mackenzie and Erickson (2004), Orlowski and Grinstein (2004), Palacin and Yoshikatsu (2004), Palmieri (2004), Verrey et al (2004) 
combined shuttles function as intercellular ammonia transporter by the transport of amino groups.

The brain depends on the supply of energy and amino acids from the systemic circulation; in addition it must be able to export waste nitrogen. Since the mitochondrial urea cycle enzymes are not expressed in brain, other pathways are used. The many transporters of the capillary endothelia (blood-brain barrier) allow a controlled uptake of amino acids into the brain and net removal of acidic and nitrogen-rich amino acids (such as glutamine). The brain capillary endothelia are spatially polarized: the $\gamma$-glutamyl cycle is expressed on the luminal side and the various amino acid transporters on the luminal and abluminal side differ as shown in Fig. 2 (Hawkins et al 2006).

Twenty per cent to fifty per cent of ammonia is taken up from capillary blood into the brain. Ammonia is considered to enter the brain by diffusion; the quantitative role of aquaporins in vivo is not clear (Marcaggi and Coles 2001). In contrast, glutamine uptake from plasma into the capillary endothelia is minor and the glutamine concentration is not really dependant on plasma glutamine concentration. Glutamine has a very low affinity for the facilitative transporter for uptake at the luminal membrane and for $\gamma$-glutamyl transpeptidase. The velocity of glutamine brain uptake in vivo at very high plasma concentrations of glutamine and low concentrations of LNAAs is not known. Glutamine in the brain is to a large extent newly synthesized in astrocytes. The transporters of the brain capillary endothelia are organized in such a way as to export glutamine and glutamate from the extracellular fluid of the brain into the capillary endothelia where glutamine is efficiently hydrolysed by glutaminase to glutamic acid. This is either exported or transaminated and the nitrogen is exported as amino acids by facilitative transporters at the luminal membrane (for further details see Fig. 2 and Hawkins et al 2006). Interestingly, Lee and colleagues (1996) and Hawkins and colleagues (2006) indicated that pyroglutamic acid (a metabolite of the $\gamma$-glutamyl cycle) stimulated the sodium-dependent amino acid transporters of the abluminal membrane of the capillary endothelia (except for the system $\mathrm{N}$ subfamily transporter). In 1986 we had found that the uptake of tryptophan was stimulated by pre-incubation of brain capillary endothelia with $10 \mathrm{mmol} / \mathrm{L} \mathrm{L}-$ or D-glutamine, but was reduced by inhibitors of $\gamma$ glutamyl transferase (acivicin) or of $\gamma$-glutamylcysteine synthase such as buthionine or methionine sulfoximine; however, we found no clue to explain the mechanism (for details see Bachmann (1992, 2002)). The stimulation of the abluminal transporters by pyroglutamic acid mentioned above could now explain our findings.

To our knowledge, the quantitative contribution of the choroid plexus (which expresses a system $\mathrm{N}$ subfamily transporter and the $\gamma$-glutamyl cycle) to the export of glutamine into the CSF has not been assessed in vivo.

The biochemical characterization of transporters and enzymes and of their compartmentation in the brain has opened many questions which need in vivo confirmation in animal models. Brain uptake of amino acids by the facilitative L1 and the cationic transporters can be measured; but the detailed fluxes in vivo in the many compartments of the brain including the capillary endothelia cannot be derived from the in vitro data determined under artificial conditions with isolated enzymes and transporters. Interactions between compartments, not only substrate concentrations, should be taken into account; additional variables interfere such as ion gradients (protons, sodium, potassium), membrane potentials, surface density of transporters and glutamatergic activity of the neurons. To our knowledge, computer modelling of fluxes has so far been restricted to the glutamine/ glutamate shuttle in the brain without taking into account the abluminal or luminal transporters of the capillary endothelia.

Consequences for interpretation of amino acids in practice

For patients with urea cycle disorders the results of amino acid concentrations in physiological fluids are mainly used for follow-up. Clinical evaluation alone does not allow control and adaptation of the treatment to the changing needs during development and the variation of intake and endogenous nitrogen-load.

In addition to the clinical signs and (dietary) history we have to evaluate the whole pattern of amino acids and also look for decreased concentrations; consider plasma ammonia concentration, electrolytes, urinary orotate and also vitamin $\mathrm{B}_{12}$ and zinc because of the restriction of natural protein; assay markers of impaired protein synthesis before it is manifest as delay of head and length growth (markers are transthyretin and coagulation factors (short half-life) and albumin or pseudocholinesterase (long half-life)); and monitor alternative-pathway drugs especially in neonates or when given at high doses.

In order to improve the medical decisions by the use of biochemical parameters, one should make sure that the results are reliable, not only by quality control of the analyses but also by clear prescription of the 
pre-analytical procedure. The samples of the analytes mentioned above should be obtained at the same time, i.e. when they are at trough level, to assure safety and avoid low concentrations being missed (and high concentrations of essential amino acids being exaggerated) by postprandial absorption or prior peroral or intravenous administration. The time delay after the end of the last meal should be at least $3.5 \mathrm{~h}(4 \mathrm{~h}$ for arginine); in practice sampling is done just preprandially. If the sampling for follow-up cannot be done at trough level, it should always be done at the same time postprandially during the absorptive phase to minimize the bias and allow comparison with previous results (taking the risk of missing the endogenous decrease of amino acid concentrations). Such results would need to be compared with reference values established during the absorptive phase of defined meals, but good reference values are lacking. We are aware that this requirement cannot always be met or that sometimes it is not feasible for practical reasons. The minimal requirement for adequate retrospective interpretation is thus that the time delay between the last intake and sampling should always be documented.

To avoid factitious hyperammonaemia the blood must be obtained without cuffing. Capillary sampling should be avoided because of the contamination from destroyed tissue and even worse by massaging. All the blood samples should be transferred to the laboratory, and centrifuged without delay and the plasma deproteinized and kept frozen at acid $\mathrm{pH}$ (column application buffer); otherwise glutamine will decrease and so will arginine (and ornithine will be formed by red cell arginase).

Glutamine in plasma does not reflect the concentrations in brain but it is an indicator of the capacity of other tissues to detoxify ammonia. Wilson et al (2001) showed that with a sufficient number of patient results there is not a good correlation between plasma ammonia and plasma glutamine concentration since the mechanisms determining their concentrations in plasma differ.

If the plasma glutamine concentration remains stable, even around the upper reference limit (Shih 2003), and if the BCAA concentrations are not below the lower reference limit and plasma ammonia not higher than three times the upper reference limit, we will not find cause for alarm. We would argue that ammonia detoxification by other pathways than the urea cycle is still adequate for the actual waste nitrogen load.

However, if glutamine concentration is increasing in plasma, the causes of increased load and/or reduced elimination should be closely investigated (Morris and Leonard 1997); excessive treatment should also be considered and the treatment strategy should be revised if necessary (Bachmann 2007).
Looking at the course and rate of increase of plasma glutamine and ammonia in comparison to the last sample(s) is more informative than comparisons with fixed upper limits for which scientific evidence is lacking (Summar 2001). Experimental research with in vivo models is needed to answer the question whether concentration patterns or the course taken by plasma concentrations of ammonia and amino acids allow prediction of imminent saturation or inhibition of critical steps of nitrogen export out of the brain and the capillary endothelia as compared with uptake (for further reading see Kanamori and Ross 2004, 2005; Kanamori et al 1998; Pichili et al 2007). Actually we recommend watching closely the course that plasma ammonia, glutamine and BCAA concentrations take.

For interpretation of low BCAA concentrations in plasma of patients with urea cycle disorders, the following causes should be considered (and might be combined): chronic or acute hyperammonaemia; excessive phenylbutyrate administration (see below); reduced dietary intake (excessive restriction of protein without adequate supplementation of essential amino acids); and infectious disease. An accurate assessment of the dietary intake is needed for determination of the causes of low BCAAs in plasma.

If not only BCAAs but also phenylalanine and tyrosine concentrations are decreased in plasma obtained preprandially, insufficient intake has to be considered. Normal or increased phenolic amino acid (and methionine) concentrations do not, however, rule out insufficient intake if there is hepatic insufficiency (fibrosis, cirrhosis).

A chronic decrease of BCAAs will likely prolong the duration of catabolism and thus the increased endogenous nitrogen load. An (increased) supplementation of BCAAs or of natural protein despite existing hyperammonaemia (usually $<250 \mu \mathrm{mol} / \mathrm{L}$ in our experience) might help to rule out BCAA deficiency as its cause. Close surveillance is needed when increasing nitrogen load in such situations to make sure that plasma ammonia decreases. We repeatedly observed in our laboratory that the plasma threonine concentration overshoots upon increasing the protein supply after excessive restriction. The mechanism leading to this increased threonine concentrations is not clear. Threonine concentration in plasma normalizes after a few days in this instance.

The 'consensus' recommendation (Summar 2001) to supply essential amino acids only in severe cases of UCD must be questioned. In our view, special essential amino acid mixtures $\left(\mathrm{UCD}^{\circledR}\right.$ (Milupa) or $\mathrm{EAM}^{\circledR}$ (SHS) for oral administration; Aminosteril $\mathrm{Hepa}^{\circledR}$ (Fresenius) for intravenous administration) 
which are enriched with BCAAs (without carnitine) should be given at least when the plasma BCAAs are decreased below the lower limit of the normal range in order to allow anabolism independently of the severity of the defect. Furthermore, supplementation of the diet with essential amino acid mixtures allows restriction of the intake of natural protein to $30-50 \%$ below RDA and thus reduction of the proportion of nonessential amino acid intake, and the nitrogen load.

\section{Alternative pathway therapy with phenylbutyrate}

Alternative pathway therapy with phenylbutyrate (or phenylacetate) further enhances the utilization of leucine in liver and kidney by increased formation of phenylbutyrylglutamine and phenylacetylglutamine, which are excreted in urine. In vivo experiments in humans, primates and rodents for assessing the anaplerosis versus cataplerosis (pyruvate dehydrogenase) have used phenylacetate to quantitatively divert $\alpha$ ketoglutarate to phenylacetylglutamine (via glutamate and glutamine), and this in absence of hyperammonaemia (Darmaun et al 1998; Hankard et al 1995; Jones et al 1998; Owen 1998). This indicates that cytosolic synthesis of glutamate (and BCAA consumption) is raised not only by increased ammonia concentration but (independently) also for compensating its utilization for phenylbutyrylglutamine or phenylacetylglutamine excretion. Scaglia and colleagues (2004, 2007) have recently shown in controls and UCD patients that upon administration of phenylbutyrate (but not of benzoate) the patients have a selective decrease of plasma BCAAs despite adequate dietary intake; however, the plasma concentrations of other essential amino acids did not change. Low BCAA concentrations published for patients treated with phenylacetate or phenylbutyrate (Maestri et al 1995, 1999) show that this is not just a theoretical or experimental consideration. The findings of Scaglia and colleagues contradict a recent report of Bhattacharya and colleagues (2007). These authors found in 18 adult OTC-deficient patients that sodium benzoate was associated with low BCAA concentrations in plasma as much as in patients treated with phenylbutyrate; in addition the patients had low phenylalanine and increased plasma concentrations of glutamine, alanine and glycine; seven patients had a vitamin $\mathrm{B}_{12}$ deficiency. Neither the diet nor the drug concentrations or doses were reported in the abstract. Thus the work needs confirmation.

We further suggest not using phenylbutyrate chronically in female patients because phenylbutyrate is a potent inhibitor of the histone deacetylases (HDAC) and impairs $\mathrm{NF \kappa B}$ activation, isoprenylation and PPRP $\alpha$ and $\gamma$ (Xin et al 1999) as shown experimentally. Its interference with cell cycle control is exploited in cancer therapy ("differentiation therapy" with anticarcinogenic drugs) (Calvaruso et al 2001; Camacho et al 2007; Chang and Szabo 2002; Lea et al 2004; Mehnert and Kelly 2007). Among the many side-effects of phenylbutyrate the most worrisome is the risk of polycystic ovaries. Wiech and colleagues reported in 1997 that the most common clinical adverse event was amenorrhoea/menstrual dysfunction: $23 \%$ of female UCD patients who had started their periods and were on phenylbutyrate therapy had irregular menstrual cycles or became amenorrhoeic (as cited by Batshaw et al 2001). To our knowledge there is no report of such clinical findings in females with urea cycle disorders who have reached postpubertal age and who were not treated with phenylbutyrate. Furthermore, this sideeffect of phenylbutyrate is listed on package inserts and should, in our view, not be taken lightly. The most likely cause appears to be histone hyperacetylation by phenylbutyrate; this same side-effect was also described for long-term valproate application (Isojärvi et al 1993). Valproate is a similar HDAC inhibitor (Blaheta and Cinatl 2002; Gottlicher 2004; Phiel et al 2001). Polycystic ovaries and hyperandrogenism were found in $80 \%$ of women on chronic valproate treatment before 20 years of age $(43 \%$ with menstrual disturbances).

Careful controlled studies are needed to assessing side-effects of long-term application of phenylbutyrate in comparison, for example, with sodium benzoate as substrates for alternative pathway therapy. Meanwhile, for reasons of safety and to avoid additional BCAA consumption, we prefer to use benzoate, which is almost as efficient as phenylbutyrate; we would add intravenous phenylacetate only during crisis situations if benzoate rose to toxic levels above $2 \mathrm{mmol} / \mathrm{L}$ (e.g. in neonates).

\section{Neurotransmitter disorders}

Extrapolation of the PKU model does not work in neurotransmitter disorders. I began to realize this when we found an increased CSF/plasma ratio of glycine $(0.31)$ in a patient with neonatal nonketotic hyperglycinaemia (NKH) (Bachmann et al 1971). Post mortem we found very high concentrations of glycine in cerebellum $(11 \mathrm{mmol} / \mathrm{kg}$ wet $\mathrm{wt}$; controls: 1.8 and 2.1) and cerebrum ( $4 \mathrm{mmol} / \mathrm{kg}$ wet $\mathrm{wt})$. Reference values of autopsied adults (mean (SEM) mmol/kg 
wet wt) are: cerebellum $2.07(0.39)$ and cortex 1.75 (0.15) (Perry et al 1971). The increases in our patient were thus 5-fold and 2-fold respectively. Our findings in $\mathrm{NKH}$ have been confirmed in vitro (Perry et al 1975) and in vivo (Novotny et al 2003). The comparison by Huisman and colleagues (2002) of in vivo (proton MRS) and ex vivo (ion-exchange chromatography) concentrations of glycine in different parts of the brain of a patient with $\mathrm{NKH}$ who died at 9 days of age is reassuring: the authors showed that the methods correlated well.

We knew in 1971 about glycinergic transmission but did not dare to use its inhibitor (strychnine) for treatment. Without success, we used means to lower glycine in plasma including benzoate. It remains open whether or when lowering glycine in plasma is useful in NKH patients. While in some patients it has been successful, in others it failed. The malfunctioning glycine cleavage system in CNS is well isolated from the peripheral circulation by the blood-brain barrier. The contribution of glycine taken up from plasma through the blood-brain barrier is considered to be minimal compared with its synthesis within the brain. In 'ketotic hyperglycinaemia' (propionic or methylmalonic acidaemia) CSF/plasma glycine ratios have been considered not to be frankly increased, despite an increase of glycine in plasma comparable to that in $\mathrm{NKH}$, if pitfalls such as valproate therapy are avoided (Applegarth and Toone 2001; Korman and Gutman 2002). Harris and colleagues reported a CSF/plasma ratio of 0.16 in a patient with propionic acidaemia (Harris et al 1981); the CSF phenylalanine or tyrosine concentrations were not reported, however, and thus blood contamination cannot be excluded. More data are needed to clarify whether the CSF/plasma ratio of glycine really allows for differentiation between nonketotic and 'ketotic' hyperglycinaemias.

In theory, lowering glycine outside of the brain by administration of high doses of sodium benzoate which is detoxified in liver to hippurate (benzoylglycine) seems not to be relevant. Still, in some patients sodium benzoate therapy has been considered to be useful. Could it be that the application of benzoate or hippurate stimulates the sodium-dependent transporters of the abluminal membrane of the brain capillary endothelia (or of astrocytes) and thus the export of extracellular glycine out of the brain?

It is now known that glycine is a neurotransmitter in strychnine-sensitive glycinergic inhibitory synapses in spinal cord and medulla as well as an excitatory coagonist in strychnine-insensitive glutamatergic (NMDA) synapses in the CNS and spinal cord. Furthermore, it is a neurotransmitter in amacrine cells of the retina (strychnine-sensitive).

Treatment should allow reduction of the effects of glycine in both the inhibitory synapses and the excitatory NMDA receptors. Treatments of NKH patients with blockers of inhibitory synapses such as strychnine or with NMDA receptor blockers have been tried separately; results were discrepant and hence nonconclusive (Gitzelmann et al 1978; Tada and Kure 2005; von Wendt et al 1980). To our knowledge the treatments have not been combined, although our understanding is that this might be tried, provided NKH has not irreversibly harmed the CNS prenatally. A delay of myelination and disturbed cytoarchitecture have been described (Bachmann et al 1971; Diezel and Martin 1966). Cerebral malformations have also been reported especially in patients with neonatal presentation (Tada and Kure 2005).

During embryonic development, glycinergic synapses are expressed early. Postnatally, the activation of the glycine receptors leads to opening of their anion channel $\left(\mathrm{Cl}^{-}\right)$and to hyperpolarization. However, during embryonic and fetal life the chloride concentration in nerve cells is high until the $\mathrm{Cl}^{-} / \mathrm{K}^{+}$cotransporter is expressed around birth. Hence in embryos the opening of the intrinsic anion channel of the glycine receptors leads to an efflux of chloride from the postsynaptic cells and thus to depolarization of target neurons by glycine (Eulenburg et al 2005). It is not clear whether this leads to prolonged excitation of postsynaptic neurons during embryonic development and to malformations in NKH.

If, after taking account of this caveat, a combined treatment by inhibitors of strychnine-sensitive glycinergic receptors and of NMDA receptors is considered, the drug effects should be monitored to assure an effective dosage. Data on glycine concentrations in autopsied cerebral or cerebellar homogenates or in vivo in patients do not allow a decision on whether treatment is effective in the synapses since concentrations in the presynaptic vesicles of glutamatergic neurons or in the synaptic cleft are thought to be physiologically 100 times higher than in brain homogenates. An average concentration of the compartments does not make sense.

In fact, we ignore the relevant pre- and postsynaptic glycine (and D-serine) concentrations in NKH patients; in addition, there might be adaptive changes of the ( $N$-glycosylated!) glycine transport proteins transporting glycine into and out of synapses and glia. Interestingly, Gly-transporter1 knockout mice show symptoms similar to NKH, while Gly-transporter2 knockouts resemble hypoglycinergic hyperekplexia (for review 
see Eulenburg et al 2005). To our knowledge, a pathophysiological role of glycine transport has not been studied in $\mathrm{N}$-glycosylation defects.

Clinical assessment of the effect of blockers like strychnine on medullar (pontine) neurons, or on amacrine cell function by electroretinography, seems feasible. Concerning the control of NMDA-blockers, Whitehead and colleagues (2004) showed by microdialysis of dorsal spine cord in rats that glycinergic coactivation led to an increase of citrulline concentration of $50 \%$ (basal value $6 \mathrm{pmol} / 20 \mu \mathrm{l}$ dialysate) as a product of NOS in the dialysate. It was measured by HPLC with fluorometric detection of the derivatized citrulline, From research in hyperammonaemic encephalopathy it is well known that glutamatergic hyperactivity leads to a calmodulin-dependent NOS activation in NMDA receptors; NO activates soluble guanylate cyclase (which can be blocked by sildenafil). One can imagine that sensitive citrulline assays in CSF might help to monitor the effects of NMDA-blockers in NKH (and urea cycle disorders?) provided the CSF concentration reflects intracellular changes as does the dialysate.

\section{Conclusions}

Awareness of basic knowledge in physiological and pathological biochemistry is needed to avoid pitfalls and appreciate the limits of interpretation imposed on us when we try to understand patterns of amino acids in plasma or other markers of intermediary metabolism. The gap between knowledge acquired in basic research on the function of isolated proteins or cells in vitro and the poor resolution and sensitivity of in vivo imaging systems indicates that translational research is needed. Actually, the advantage of localization by quantitative in vivo imaging methods is compromised by the brain sample volumes of millilitres and by the low sensitivity limited to quantifying solute concentration, usually above $0.1 \mathrm{mmol} / \mathrm{L}$. Such samples are equivalent to homogenates of different structures, cell types and organelles. They are useful for diagnoses of gross changes, despite averaging of concentrations differences, which can be 1000-fold between compartments. To bridge this gap, coordinated in vitro, ex vivo and in vivo research on relevant pathways is needed: e.g. quantification coupled to localization of metabolites, RNA and protein expression at tissue and cellular level from mixed brain cell culture systems or ex vivo from experimental animals with defined gene defects and feeding conditions. The goal would be a functional anatomy and cytology at high sensitivity and high resolution. Such results could be compared for validation with in vivo investigations on patients and controls for defined clinical problems in inherited diseases of intermediary metabolism. Careful planning with accepted protocols and collection of information in international registries with predefined minimal information on patients and their data would help to concentrate the efforts on these rare disorders with much interindividual variance at all levels. The hope is that proceeding in a concerted way (e.g. within the SSIEM) will result in more effective prevention and treatment of inborn errors of metabolism, to the benefit of the patients.

\section{References}

Appel SH (1966) Inhibition of brain protein synthesis: an approach to the biochemical basis of neurological dysfunction in the amino-acidurias. Trans N Y Acad Sci 29: 63-70.

Applegarth DA, Toone JR (2001) Nonketotic hyperglycinemia (glycine encephalopathy): laboratory diagnosis. Mol Genet Metab 74: 139-146.

Bachmann C (1992) Ornithine carbamoyl transferase deficiency: findings, models and problems. $J$ Inherit Metab Dis 15: $578-591$.

Bachmann C (2002) Mechanisms of hyperammonemia. Clin Chem Lab Med 40: 653-662.

Bachmann C (2007) Hyperammonemia: Review of current treatment strategies. In: Bachmann C, Häberle J, Leonard J, eds. Pathophysiology and Management of Hyperammonaemia. Heilbronn: SPS Verlagsgesellschaft, 157-173.

Bachmann C, Mihatsch MJ, Baumgartner RE et al (1971) Nonketotic hyperglycinemia: peracute course in neonatal period. Helv Paediatr Acta 26: 228-243.

Bak LK, Schousboe A, Waagepetersen HS (2006) The glutamate/ GABA-glutamine cycle: aspects of transport, neurotransmitter homeostasis and ammonia transfer. J Neurochem 98: 641-653.

Batshaw ML, MacArthur RB, Tuchman M (2001) Alternative pathway therapy for urea cycle disorders: twenty years later. $J$ Pediatr 138: S46-54; discussion S54-45.

Berger V, Larondelle Y, Trouet A, Schneider YJ (2000) Transport mechanisms of the large neutral amino acid 1-phenylalanine in the human intestinal epithelial Caco-2 cell line. J Nutr 130: 2780-2788.

Bhattacharya K, Briddon A, Lee P (2007) A review of biochemical outcomes of adults with OTC deficiency. $J$ Inherit Metab Dis 30: 82.

Bixel MG, Shimomura Y, Hutson SM, Hamprecht B (2001) Distribution of key enzymes of branched-chain amino acid metabolism in glial and neuronal cells in culture. J Histochem Cytochem 49: 407-418.

Blaheta RA, Cinatl J Jr (2002) Anti-tumor mechanisms of valproate: a novel role for an old drug. Med Res Rev 22: 492-511.

Brosnan JT, Brosnan ME (2006) Branched-chain amino acids: enzyme and substrate regulation. J Nutr 136: 207-211.

Bruhat A, Jousse C, Fafournoux P (1999) Amino acid limitation regulates gene expression. Proc Nutr Soc 58: 625-632.

Calvaruso G, Carabillo M, Giuliano M et al (2001) Sodium phenylbutyrate induces apoptosis in human retinoblastoma 
Y79 cells: the effect of combined treatment with the topoisomerase I-inhibitor topotecan. Int J Oncol 18: 1233-1237.

Camacho LH, Olson J, Tong WP, Young CW, Spriggs DR, Malkin MG (2007) Phase I dose escalation clinical trial of phenylbutyrate sodium administered twice daily to patients with advanced solid tumors. Investigational New Drugs 25: 131-138.

Chang TH, Szabo E (2002) Enhanced growth inhibition by combination differentiation therapy with ligands of peroxisome proliferator-activated receptor-gamma and inhibitors of histone deacetylase in adenocarcinoma of the lung. Clin Cancer Res 8: 1206-1212.

Chang TW, Goldberg AL (1978) The origin of alanine produced in skeletal muscle. J Biol Chem 253: 3677-3684.

Chen N, Reith M, Quick M (2004) Synaptic uptake and beyond: the sodium- and chloride dependent neurotransmitter transporter family SLC6. Pflügers Arch Eur J Physiol 447: $519-531$.

Codogno P, Meijer AJ (2005) Autophagy and signaling: their role in cell survival and cell death. Cell Death Differ 12: $1509-1518$.

Curthoys NP, Watford M (1995) Regulation of glutaminase activity and glutamine metabolism. Annu Rev Nutr 15: 133-159.

Darmaun D, Welch S, Rini A, Sager BK, Altomare A, Haymond MW (1998) Phenylbutyrate-induced glutamine depletion in humans: effect on leucine metabolism. Am J Physiol Endocrinol Metab 274: 801-807.

Diezel PB, Martin K (1966) Hyperglycinemia (glycinosis) with familial idiopathic hyperglycinuria. 1st observation in Germany. Dtsch Med Wochenschr 91: 2249-2254.

Dingemanse MA, De Jonge WJ, De Boer PAJ, Mori M, Lamers WH, Moorman AF (1996) Development of the ornithine cycle in rat liver: Zonation of a metabolic pathway. Hepatology 24: 407-411.

Endo Y, Fu Z, Abe K, Arai S, Kato H (2002) Dietary protein quantity and quality affect rat hepatic gene expression 1 . J Nutr 132: 3632-3637.

Eulenburg V, Armsen W, Betz H, Gomeza J (2005) Glycine transporters: essential regulators of neurotransmission. Trends Biochem Sci 30: 325-333.

Gebhardt R, Baldysiak-Figiel A, Krugel V, Ueberham E, Gaunitz F (2007) Hepatocellular expression of glutamine synthetase: An indicator of morphogen actions as master regulators of zonation in adult liver. Prog Histochem Cytochem 41: 201-266.

Gitzelmann R, Steinmann B, Otten A et al (1978) Nonketotic hyperglycinemia treated with strychnine, a glycine receptor antagonist. Helv Paediatr Acta 32: 517-525.

Gottlicher M (2004) Valproic acid: an old drug newly discovered as inhibitor of histone deacetylases. Ann Hemat 83(Supplement 1): S91-92.

Güttler F, Lou H (1986) Dietary problems of phenylketonuria: effect on CNS transmitters and their possible role in behaviour and neuropsychological function. $J$ Inherit Metab Dis 9: 169-177.

Hall TR, Wallin R, Reinhart GD, Hutson SM (1993) Branched chain aminotransferase isoenzymes. Purification and characterization of the rat brain isoenzyme. $J$ Biol Chem 268: 3092-3098.

Hankard RG, Darmaun D, Sager BK, D'Amore D, Parsons WR, Haymond M (1995) Response of glutamine metabolism to exogenous glutamine in humans. Am J Physiol Endocrinol Metab 269: 663-670.

Harris DJ, Thompson RM, Wolf B, Yang BI (1981) Propionyl coenzyme A carboxylase deficiency presenting as nonketotic hyperglycinaemia. J Med Genet 18: 156-157.
Harris TE, Chi A, Shabanowitz J, Hunt DF, Rhoads RE, Lawrence JC Jr (2006) mTOR-dependent stimulation of the association of eIF4G and eIF3 by insulin. EMBO $J \mathbf{2 5}$ : $1659-1668$.

Häussinger D (1983) Hepatocyte heterogeneity in glutamine and ammonia metabolism and the role of an intercellular glutamine cycle during ureogenesis in perfused rat liver. Eur J Biochem 133: 269-275.

Hawkins RA, O'Kane RL, Simpson IA, Vina JR (2006) Structure of the blood-brain barrier and its role in the transport of amino acids. $J$ Nutr 136: 218-226.

Huisman T, Thiel T, Steinmann B, Zeilinger G, Martin E (2002) Proton magnetic resonance spectroscopy of the brain of a neonate with nonketotic hyperglycinemia: in vivo-in vitro (ex vivo) correlation. Eur Radiol 12: 858-861.

Hutson SM, Berkich D, Drown P, Xu B, Aschner M, LaNoue KF (1998) Role of branched-chain aminotransferase isoenzymes and gabapentin in neurotransmitter metabolism. J Neurochem 71: 863-874.

Hutson SM, Lieth E, LaNoue KF (2001) Function of leucine in excitatory neurotransmitter metabolism in the central nervous system. $J$ Nutr 131: 846-850.

Hutson SM, Wallin R, Hall TR (1992) Identification of mitochondrial branched chain aminotransferase and its isoforms in rat tissues. $J$ Biol Chem 267: 15681-15686.

Islam M, Wallin R, Wynn RM et al (2007) A novel branchedchain amino acid metabolon: protein-protein interactions in a supramolecular complex. J Biol Chem 282: 11893-11903.

Isojärvi J, Laatikainen TJ, Pakarinen AJ, Juntunen K, Myllylä VV (1993) Polycystic ovaries and hyperandrogenism in women taking valproate for epilepsy. $N$ Engl J Med 329: $1383-1388$.

Jones JG, Solomon MA, Sherry AD, Jeffrey FMH, Malloy CR (1998) 13C NMR measurements of human gluconeogenic fluxes after ingestion of $\left[\mathrm{U}-{ }^{13} \mathrm{C}\right]$ propionate, phenylacetate, and acetaminophen. Am J Physiol Endocrinol Metab 275: 843-852.

Jousse C, Bruhat A, Ferrara M, Fafournoux P (1998) Physiological concentration of amino acids regulates insulin-like-growthfactor-binding protein 1 expression. Biochem J 334: 147-153.

Jungermann K, Kietzmann T (1996) Zonation of parenchymal and nonparenchymal metabolism in liver. Annu Rev Nutr 16: 179-203.

Kanai Y, Hediger MA (2004) The glutamate/neutral amino acid transporter family SLC1: molecular, physiological and pharmacological aspects. Pflügers Arch Eur J Physiol 447: 469-479.

Kanamori K, Ross BD (2004) Quantitative determination of extracellular glutamine concentration in rat brain, and its elevation in vivo by system A transport inhibitor, alpha(methylamino) isobutyrate. J Neurochem 90: 203-210.

Kanamori K, Ross BD (2005) Suppression of glial glutamine release to the extracellular fluid studied in vivo by NMR and microdialysis in hyperammonemic rat brain. $J$ Neurochem 94: 74-85.

Kanamori K, Ross BD, Kondrat RW (1998) Rate of glutamate synthesis from leucine in rat brain measured in vivo by $15 \mathrm{~N}$ NMR. J Neurochem 70: 1304-1315.

Koch R, Moseley KD, Yano S, Nelson Jr M, Moats RA (2003) Large neutral amino acid therapy and phenylketonuria: a promising approach to treatment. Mol Genet Metab 79: 110-113.

Korman SH, Gutman A (2002) Pitfalls in the diagnosis of glycine encephalopathy (non-ketotic hyperglycinemia). Dev Med Child Neurol 44: 712-720.

Labow BI, Souba WW, Abcouwer SF (2001) Mechanisms governing the expression of the enzymes of glutamine 
metabolism-glutaminase and glutamine synthetase. J Nutr 131: 2467S-2474S; discussion 2486S-2467S.

Lea MA, Sura M, Desbordes C (2004) Inhibition of cell proliferation by potential peroxisome proliferator-activated receptor (PPAR) gamma agonists and antagonists. Anticancer Res 24: 2765-2771.

Lee WJ, Hawkins RA, Peterson DR, Vina JR (1996) Role of oxoproline in the regulation of neutral amino acid transport across the blood-brain barrier. J Biol Chem 271: 19129.

Leweling H, Breitkreutz R, Behne F, Staedt U, Striebel JP, Holm E (1996) Hyperammonemia-induced depletion of glutamate and branched-chain amino acids in muscle and plasma. J Hepatol 25: 756-762.

Lou HC, Güttler F, Lykkelund C, Bruhn P, Niederwieser A (1985) Decreased vigilance and neurotransmitter synthesis after discontinuation of dietary treatment for phenylketonuria in adolescents. Eur J Pediatr 144: 17-20.

Mackenzie B, Erickson JD (2004) Sodium-coupled neutral amino acid (System N/A) transporters of the SLC38 gene family. Pflügers Arch Eur J Physiol 447: 784-795.

Maestri NE, Clissold DB, Brusilow SW (1995) Long-term survival of patients with argininosuccinate synthetase deficiency. J Pediatr 127: 929-935.

Maestri NE, Clissold D, Brusilow SW (1999) Neonatal onset ornithine transcarbamylase deficiency: a retrospective analysis. J Pediatr 134: 268-272.

Marcaggi P, Coles JA (2001) Ammonium in nervous tissue: transport across cell membranes, fluxes from neurons to glial cells, and role in signalling. Prog Neurobiol 64: 157-183.

Matalon R, Surendran S, Matalon KM et al (2003) Future role of large neutral amino acids in transport of phenylalanine into the brain. Pediatr 112: 1570-1574.

Matalon R, Michals-Matalon K, Bhatia G et al (2006) Large neutral amino acids in the treatment of phenylketonuria (PKU). J Inherit Metab Dis 29: 732-738.

Matalon R, Michals-Matalon K, Bhatia G et al (2007) Double blind placebo control trial of large neutral amino acids in treatment of PKU: Effect on blood phenylalanine. $J$ Inherit Metab Dis 30: 153-158.

Mehnert JM, Kelly WK (2007) Histone deacetylase inhibitors: biology and mechanism of action. Cancer J 13: 23-29.

Meijer AJ, Dubbelhuis PF (2004) Amino acid signalling and the integration of metabolism. Biochem Biophys Res Commun 313: $397-403$

Miller LP, Pardridge WM, Braun LD, Oldendorf WH (1985) Kinetic constants for blood-brain barrier amino acid transport in conscious rats. $J$ Neurochem 45: 1427-1432.

Miyanaka K, Gotoh T, Nagasaki A et al (1998) Immunohistochemical localization of arginase II and other enzymes of arginine metabolism in rat kidney and liver. Histochem J 30: 741-751.

Morris AAM, Leonard JV (1997) Early recognition of metabolic decompensation. Arch Dis Child 76: 555-556.

Nishitani S, Ijichi C, Takehana K, Fujitani S, Sonaka I (2004) Pharmacological activities of branched-chain amino acids: specificity of tissue and signal transduction. Biochem Biophys Res Commun 313: 387-389.

Nissim I (1999) Newer aspects of glutamine/glutamate metabolism: the role of acute pH changes. Am J Physiol 277: 493-497.

Novotny EJ, Fulbright RK, Pearl PL, Gibson KM, Rothman DL (2003) Magnetic resonance spectroscopy of neurotransmitters in human brain. Ann Neurol 54: S25-S31.

Oldendorf WH, Sisson BW, Silverstein A (1971) Brain uptake of selenomethionine Se 75. II. Reduced brain uptake of selenomethionine Se 75 in phenylketonuria. Arch Neurol 24: $524-528$
Orlowski J, Grinstein S (2004) Diversity of the mammalian sodium/proton exchanger SLC9 gene family. Pflügers Arch Eur J Physiol 447: 549-565.

Owen OE (1998) Protein, fat, and carbohydrate requirements during starvation: anaplerosis and cataplerosis]. J Nutr 68 : 12-34.

Palacin M, Yoshikatsu K (2004) The ancillary proteins of HATs: SLC3 family of amino acid transporters. Pflügers Arch Eur J Physiol 447: 490-494.

Palmieri F (2004) The mitochondrial transporter family (SLC25): physiological and pathological implications. Pflügers Arch Eur J Physiol 447: 689-709.

Perry TL, Hansen S, Berry K, Mok C, Lesk D (1971) Free amino acids and related compounds in biopsies of human brain. J Neurochem 18: 521-528.

Perry TL, Urquhart N, MacLean J et al (1975) Nonketotic hyperglycinemia. Glycine accumulation due to absence of glycerine cleavage in brain. $N$ Engl J Med 292: 1269-1273.

Phiel CJ, Zhang F, Huang EY, Guenther MG, Lazar MA, Klein PS (2001) Histone deacetylase is a direct target of valproic acid, a potent anticonvulsant, mood stabilizer, and teratogen. J Biol Chem 276: 36734-36741.

Pichili VB, Rao KV, Jayakumar AR, Norenberg MD (2007) Inhibition of glutamine transport into mitochondria protects astrocytes from ammonia toxicity. Glia 55: 801-809.

Pietz J, Kreis R, Rupp A et al (1999) Large neutral amino acids block phenylalanine transport into brain tissue in patients with phenylketonuria. J Clin Invest 103: 1169-1178.

Plaitakis A, Spanaki C, Mastorodemos V, Zaganas I (2003) Study of structure-function relationships in human glutamate dehydrogenases reveals novel molecular mechanisms for the regulation of the nerve tissue-specific (GLUD2) isoenzyme. Neurochem Int 43: 401-410.

Proud CG (2004a) mTOR-mediated regulation of translation factors by amino acids. Biochem Biophys Res Commun 313: $429-436$.

Proud CG (2004b) Role of mTOR signalling in the control of translation initiation and elongation by nutrients. Curr Top Microbiol Immunol 279: 215-244.

Rennie MJ, Wackerhage H, Spangenburg EE, Booth FW (2004) Control of the size of the human muscle mass. Ann Rev Physiol 66: 799-828.

Scaglia F, Carter S, O'Brien WE, Lee B (2004) Effect of alternative pathway therapy on branched chain amino acid metabolism in urea cycle disorder patients. Mol Genet Metab 81: S79-85.

Scaglia F, Lanpher B, Marinim J, Lee B (2007) Role of branched chain amino acids in patients with urea cycle disorders. In: Bachmann C, Häberle J, Leonard J, eds. Pathophysiology and Management of Hyperammonaemia. Heilbronn: SPS Verlagsgesellschaft, 157-173.

Shih V (2003) Amino acid analysis. In: Blau N, Duran M, Blaskovics M, Gibson M, eds. Physician's Guide to the Laboratory Diagnosis of Metabolic Diseases. Berlin: Springer-Verlag, 11-26.

Summar M (2001) Current strategies for the management of neonatal urea cycle disorders. J Pediatr 138: S30-39.

Sweatt AJ, Garcia-Espinosa MA, Wallin R, Hutson SM (2004) Branched-chain amino acids and neurotransmitter metabolism: Expression of cytosolic branched-chain aminotransferase(BCATc) in the cerebellum and hippocampus. $J$ Comp Neurol 477: 360-370.

Tada K, Kure S (2005) Nonketotic hyperglycinemia: Pathophysiological studies. Proc Japan Acad 81: 411-417.

Taub F, Johnson TC (1975) The mechanism of polyribosome disaggregation in brain tissue by phenylalanine. Biochem $J$ 151: $173-180$. 
Verrey F, Closs EI, Wagner CA, Palacin M, Endou H, Kanai Y (2004) CATs and HATs: the SLC7 family of amino acid transporters. Pflügers Arch Eur J Physiol 447: 532-542.

von Wendt L, Simila S, Saukkonen AL, Koivisto M (1980) Failure of strychnine treatment during the neonatal period in three Finnish children with nonketotic hyperglycinemia. Pediatrics 65: 1166-1169.

Welbourne TC (1987) Interorgan glutamine flux in metabolic acidosis. Am J Physiol 253: F1069-1076.

Whitehead KJ, Pearce SM, Walker G, Sundaram H, Hill D, Bowery NG (2004) Positive $N$-methyl-D-aspartate receptor modulation by selective glycine transporter-1 inhibition in the rat dorsal spinal cord in vivo. Neuroscience 126: 381-390.
Wilson CJ, Lee PJ, Leonard JV (2001) Plasma glutamine and ammonia concentrations in ornithine carbamoyltransferase deficiency and citrullinaemia. J Inherit Metab Dis 24: 691695.

Xin X, Yang S, Kowalski J, Gerritsen ME (1999) Peroxisome proliferator-activated receptor gamma ligands are potent inhibitors of angiogenesis in vitro and in vivo. J Biol Chem 274: 9116-9121.

Yoshizawa F (2004) Regulation of protein synthesis by branched-chain amino acids in vivo. Biochem Biophys Res Commun 313: 417-422.

Yudkoff M, Daikhin Y, Nissim I et al (2005) Brain amino acid requirements and toxicity: the example of leucine. JNutr 135: $1531-1538$. 